\title{
Plasmonic Metasurfaces Based on Pyramidal Nanoholes for High- Efficiency SERS Biosensing
}

\author{
Giovanna Palermo, ${ }^{\nabla}$ Massimo Rippa, ${ }^{\nabla}$ Ylli Conti, Ambra Vestri, Riccardo Castagna, Giovanna Fusco,
} Elisabetta Suffredini, Jun Zhou, Joseph Zyss, Antonio De Luca,* and Lucia Petti*

Cite This: ACS Appl. Mater. Interfaces 2021, 13, 43715-43725

Read Online
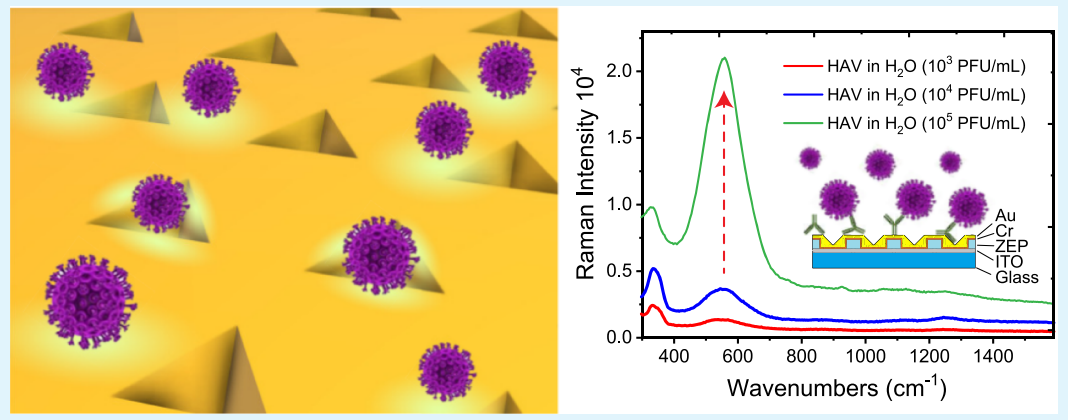

ABSTRACT: An inverted pyramidal metasurface was designed, fabricated, and studied at the nanoscale level for the development of a label-free pathogen detection on a chip platform that merges nanotechnology and surface-enhanced Raman scattering (SERS). Based on the integration and synergy of these ingredients, a virus immunoassay was proposed as a relevant proof of concept for very sensitive detection of hepatitis A virus, for the first time to our best knowledge, in a very small volume $(2 \mu \mathrm{L})$, without complex signal amplification, allowing to detect a minimal virus concentration of $13 \mathrm{pg} / \mathrm{mL}$. The proposed work aims to develop a high-flux and high-accuracy surface-enhanced Raman spectroscopy (SERS) nanobiosensor for the detection of pathogens to provide an effective method for early and easy water monitoring, which can be fast and convenient.

KEYWORDS: surface-enhanced Raman scattering, metasurface, plasmonics, biosensing, pyramidal nanoholes, hepatitis A virus

\section{INTRODUCTION}

Detection of pathogens present in food and water is essential to help ensure food safety. In recent years, the progress of technology and applications of plasmonic nanomaterials, as well as surface-enhanced Raman scattering (SERS) effect, allowed developing an effective method to achieve high flux, high accuracy, and high sensitivity for analytical purposes and pathogen detection. ${ }^{1-5}$ Furthermore, the last decade has been characterized by the development of artificial materials

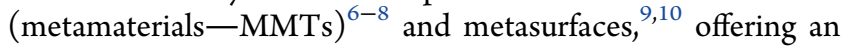
advanced and unprecedented control of the interaction between the electromagnetic (EM) field and biological matter, thereby enabling the design of increasingly innovative nanosensors. ${ }^{11-17}$ Plasmonic technologies represent a rapidly growing field that allow to develop valid alternatives to conventional diagnostic methods. Virus diagnosis is mainly carried out using various molecular techniques based on polymerase chain reaction (PCR), in situ hybridization process, as well as enzyme-linked immunosorbent assay (ELISA) ${ }^{18}$ Although these methods have many advantages and are widely used, they face some limitations. Specifically, they require multiple sampling tests and reagents, complicated multistep procedures, and highly specialized staff, entailing high costs. Moreover, the time-consuming nature and/or the need for sophisticated instrumentation of those methods limit their on-site applications. It is, therefore, necessary to identify new analytical routes, which are at the same time sensitive, specific, have short execution times, and can be used in the screening analysis of water samples and as an alternative to conventional methods. Considerable progress has been made in the past 5-10 years in the development of plasmonic nanobiosensors for detection of viruses, ${ }^{19-26}$ and the possibility to discriminate different viruses, and even different strains of the same virus, by SERS fingerprint has already been successfully reported in the literature. ${ }^{27-29}$

In this context, hepatitis A virus (HAV), belonging to the Picornaviridae family, stands out as a waterborne and foodborne human pathogen, responsible for about half of the

Received: July 3, 2021

Accepted: July 29, 2021

Published: September 1, 2021 

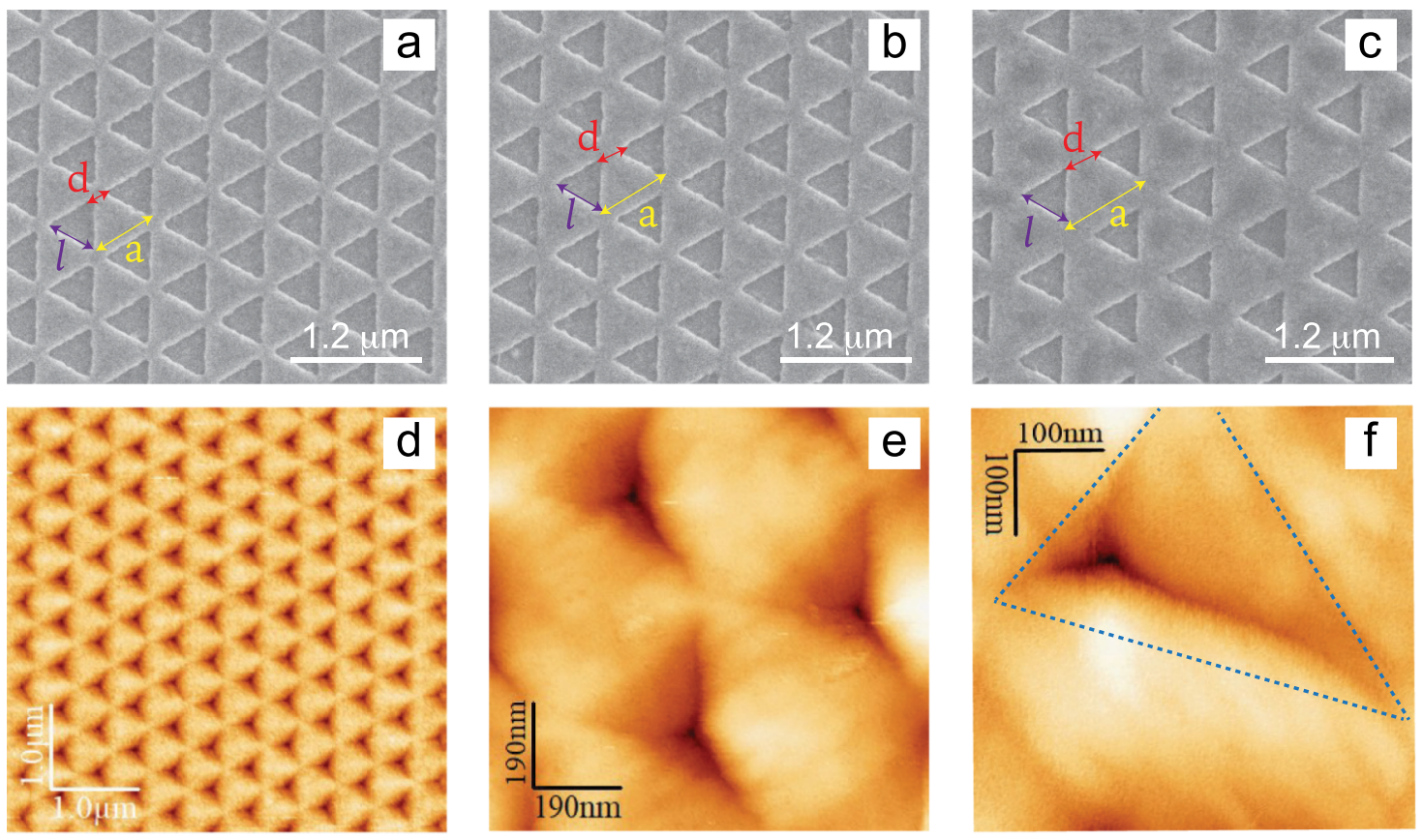

Figure 1. Scanning electron microscopy (SEM) images of Au nanopyramid samples: (a) A1 (d=110 nm, $a=500 \mathrm{~nm}),(\mathrm{b}) \mathrm{B} 1(d=160 \mathrm{~nm}, a=$ $550 \mathrm{~nm})$, and $(\mathrm{c}) \mathrm{C} 1(d=250 \mathrm{~nm}, a=640 \mathrm{~nm})$. The side: $l=390 \mathrm{~nm}$. White bar is $1.2 \mu \mathrm{m}$. Atomic force microscopy (AFM) images with different magnification of sample A1: (d) $5 \times 5 \mu \mathrm{m}^{2}$, (e) $1 \times 1 \mu \mathrm{m}^{2}$, and (f) $500 \times 500 \mathrm{~nm}^{2}$.

total number of hepatitis infections diagnosed worldwide. ${ }^{30-36}$ To date, there are very few alternative methods stated in the literature based mainly on electrochemical and optical biosensors for HAV detection. ${ }^{37,38}$

Plasmonic properties of metallic nanoparticles have been used to detect viruses with different approaches; ${ }^{28}$ however, there seems to be a lack of studies concerning plasmonic devices for the direct detection of the whole HAV. In 2002, Cao et al. developed a system based on gold nanoparticles to detect HAV nucleic acids using a SERS spectroscopy method. ${ }^{39}$ In 2009, Jang et al. reported a restriction-enzymecoded DNA detection method based on gold nanoparticle probes for the HAV DNA target. ${ }^{40}$ In 2014, Liu et al. developed a one-tube colorimetric platform based on LSPRactive nanoparticles sensitive to oligonucleotides related to a specific HAV gene. ${ }^{41}$ In 2017, Su et al. used a method based on DNA-mediated gold-silver nanoparticles for the simultaneous SERS detection of various targets, including HAV nucleic acids. $^{42}$

Our work aims at introducing innovative engineered metasurfaces that resulted in highly sensitive, selective, and reliable pathogen detection methods. Herein, we report on the engineering of a novel plasmonic metasurface based on inversed pyramidal nanoholes (P-NHs $)^{43-49}$ that can be used as a plasmonic substrate for sensitive SERS analysis ${ }^{1-4}$ and tested, as a proof of concept, for the analysis and detection of HAV in water. The considered P-NH pattern, based on a periodic geometry of nanocavities, presents some important features as compared to other patterns reported in the literature. In comparison to other patterns based on nanoholes (where no gold is present in the holes), our nanostructure consists of a real continuous metal layer with a plasmonic material present also in the cavities $(\mathrm{FF}=1)$. This last property (i) maximizes the area available for functionalization, (ii) makes the plasmonic field active for sensing also inside the cavities, and (iii) allows to reduce the fluorescence signal generated by both the layers below the surface and by the glass substrate. The P-NH nanopattern that we propose is hexagonal based. Such arrangement enables a higher packing factor of the unit cell and so, combined with its smaller dimensions, a higher density of cells (and therefore of hot spots) compared to commercial substrates, such as those belonging to the Klarite family. ${ }^{45-47}$ It is worthwhile to underline that this difference is important in terms of hot spot intensity. In fact, the vertices of the triangular base of the proposed pyramid (characterized by an angular aperture of $60^{\circ}$ ) provide a higher-intensity hot spot than those of the square base (angular aperture $90^{\circ}$ ) of the Klarite substrates. Moreover, the lower area of our pyramids $\left(6.59 \times 10^{-8} \mathrm{~mm}^{2}\right)$ than the pyramids of the Klarite substrates $\left(2.16 \times 10^{-6} \mathrm{~mm}^{2}\right.$ for the standard Klarite 302 and $2.16 \times 10^{-6}$ $\mathrm{mm}^{2}$ for the next-generation Klarite 308) favors virus detection, as reported in this study. We studied the geometries with different interdistances among the P-NHs by means of simulations based on a finite element method (FEM) and fabricated them with the electron beam lithography (EBL) technique. Realized nanostructures and metasurfaces were morphologically characterized using scanning electron microscopy (SEM) and atomic force microscopy (AFM) techniques and analyzed in near and far fields using scanning near-field optical microscopy (SNOM) and vis-NIR spectroscopy, respectively. As a result, we demonstrated the possibility to achieve the SERS fingerprint of the physioabsorbed HAV in water with an extremely low sample volume $(2 \mu \mathrm{L})$. It is worthwhile to underline that it was chosen to detect HAV in water since enteric viruses are normally present in aquatic environments. ${ }^{50,51}$ As a proof of concept, we also functionalized our metasurface using a HAV antibody to prove that our engineered substrate was suitable for the development of a biosensor exploitable for the quantitative detection of HAV by SERS. In particular, we used the biofunctionalized metasurface to carry out SERS measurements in the presence of various $\mathrm{HAV}$ concentrations to detect a minimum quantity of $13 \mathrm{pg} /$ 

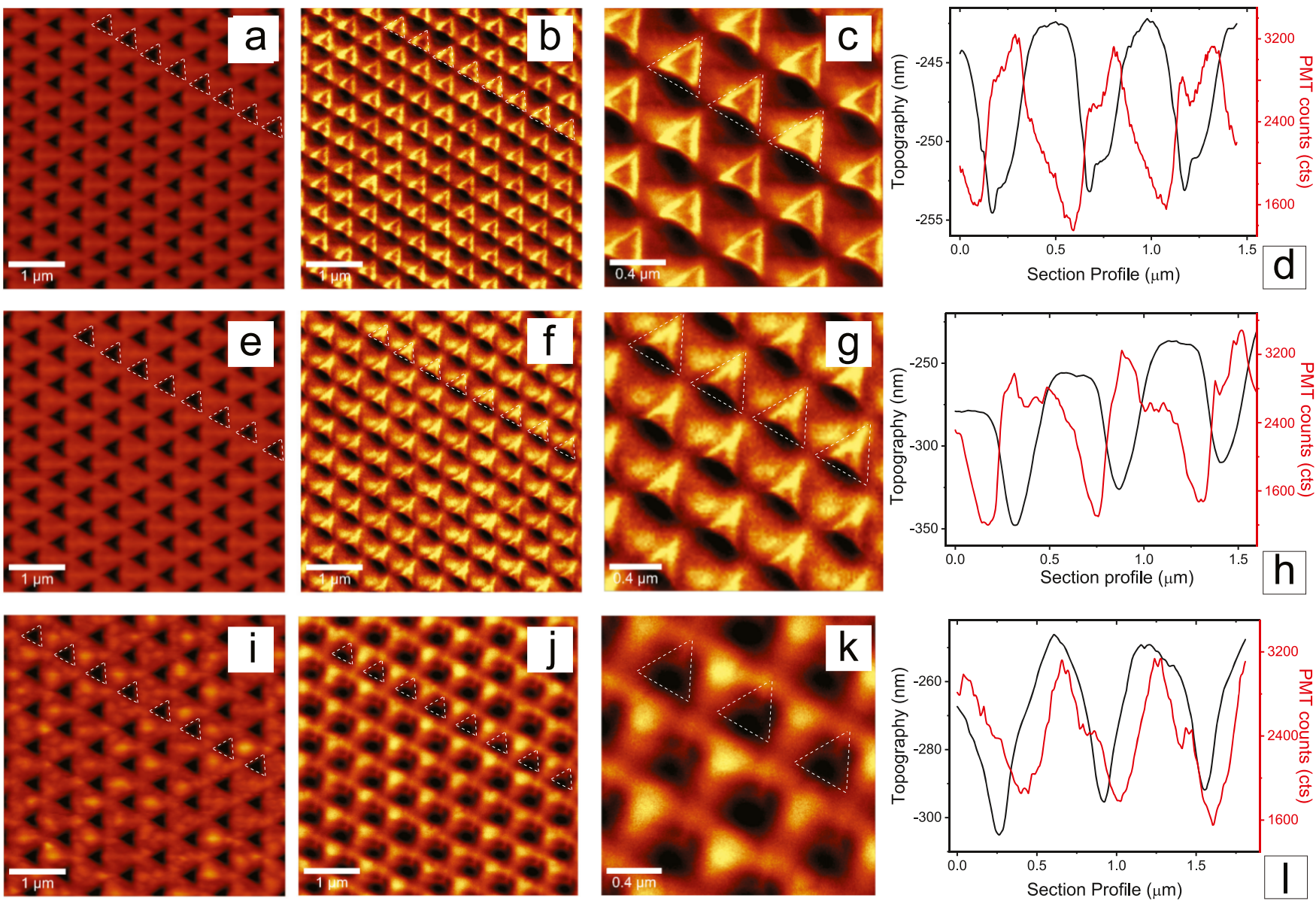

Figure 2. Polarization-dependent near-field response of Au nanopyramid samples. (a) Topography and (b, c) the corresponding SNOM images with (d) line profiles of topography and SNOM images along the white line from (a) and (b) of sample A1, when excited by a $45^{\circ}$ polarized 532 $\mathrm{nm}$ CW laser beam. (e) Topography and ( $\mathrm{f}, \mathrm{g}$ ) the corresponding SNOM images with (h) line profiles of topography and SNOM images along the white line from (e) and (f) of sample B1, when excited by a $45^{\circ}$ polarized $532 \mathrm{~nm}$ laser beam. (i) Topography and (j, k) the corresponding SNOM images with (1) line profiles of topography and SNOM images along the white line from (i) and (j) of sample $\mathrm{C} 1$, when excited by a $45^{\circ}$ polarized $532 \mathrm{~nm}$ laser beam.

mL. A different virus, Murine Norovirus (MuNoV), was finally used to preliminarily evaluate the specificity of our immunosurface. Our immuno approach allows to detect the whole virus without any need of nucleic acid extraction steps, thus making it feasible for both fast and easy detection and in situ analysis with portable systems. This is the first time, to our best knowledge, that a plasmonic engineered substrate is used for both the SERS fingerprint identification and the immunoSERS detection of HAV.

\section{RESULTS AND DISCUSSION}

Metasurfaces operating in the frequency band of the visible range have been fabricated based on gold inverted pyramidal nanoholes (P-NHs) using a high-resolution electron beam lithography (EBL) process (see the Materials and Methods section for details). The fabricated samples, characterized by a hexagonal arrangement of the P-NHs and different geometrical parameters, are identified as Al (edge-edge interdistance $d=$ $110 \mathrm{~nm}$, red arrow in Figure 1a, period $a=500 \mathrm{~nm}$, yellow arrow in Figure 1a), B1 $(d=160 \mathrm{~nm}, a=550 \mathrm{~nm})$, and C1 $(d=$ $250 \mathrm{~nm}, a=640 \mathrm{~nm}$ ). The side $l$ of the triangular base is fixed to $390 \mathrm{~nm}$ for all of the three samples.

The surface analysis of all of the fabricated samples has been carried out using a scanning electron microscope (SEM) and an atomic force microscope (AFM). The SEM analysis is reported in Figure $1 \mathrm{a}-\mathrm{c}$ for the samples $\mathrm{A} 1, \mathrm{~B} 1$, and $\mathrm{C} 1$, respectively. They provide evidence for the high uniformity and regularity of the triangular geometries over the whole patterned area. The resulting inverted pyramidal shape of the nanostructures is evidenced by the AFM analysis, which enables to access the wedge profile of the pyramid on its three sides. In particular, we report the AFM images with different magnifications of the sample (see Figure 1d,e). In the Supporting Information, a cut of the AFM image is reported (Figure S1), evidencing a ca. $50 \mathrm{~nm}$ deep inverted pyramidal shape.

The transmitted field has been acquired in the far field by a spectrometer (see the Materials and Methods section) in the visible range to study the characteristic plasmonic modes of the three metasurfaces. Extinction spectra, reported in the Supporting Information (Figure S2), show a predominant absorbance peak around $532 \mathrm{~nm}$, typical of gold, whereas other two peaks around 650 and $750 \mathrm{~nm}$ are mainly related to the coupling between the nanocavities. By increasing the interdistance among them, a red shift of these secondary resonances occurs (see Figure S2 in the Supporting Information). To ascertain the nature of these modes, a numerical analysis with Comsol Multiphysics has been conducted. In particular, the 

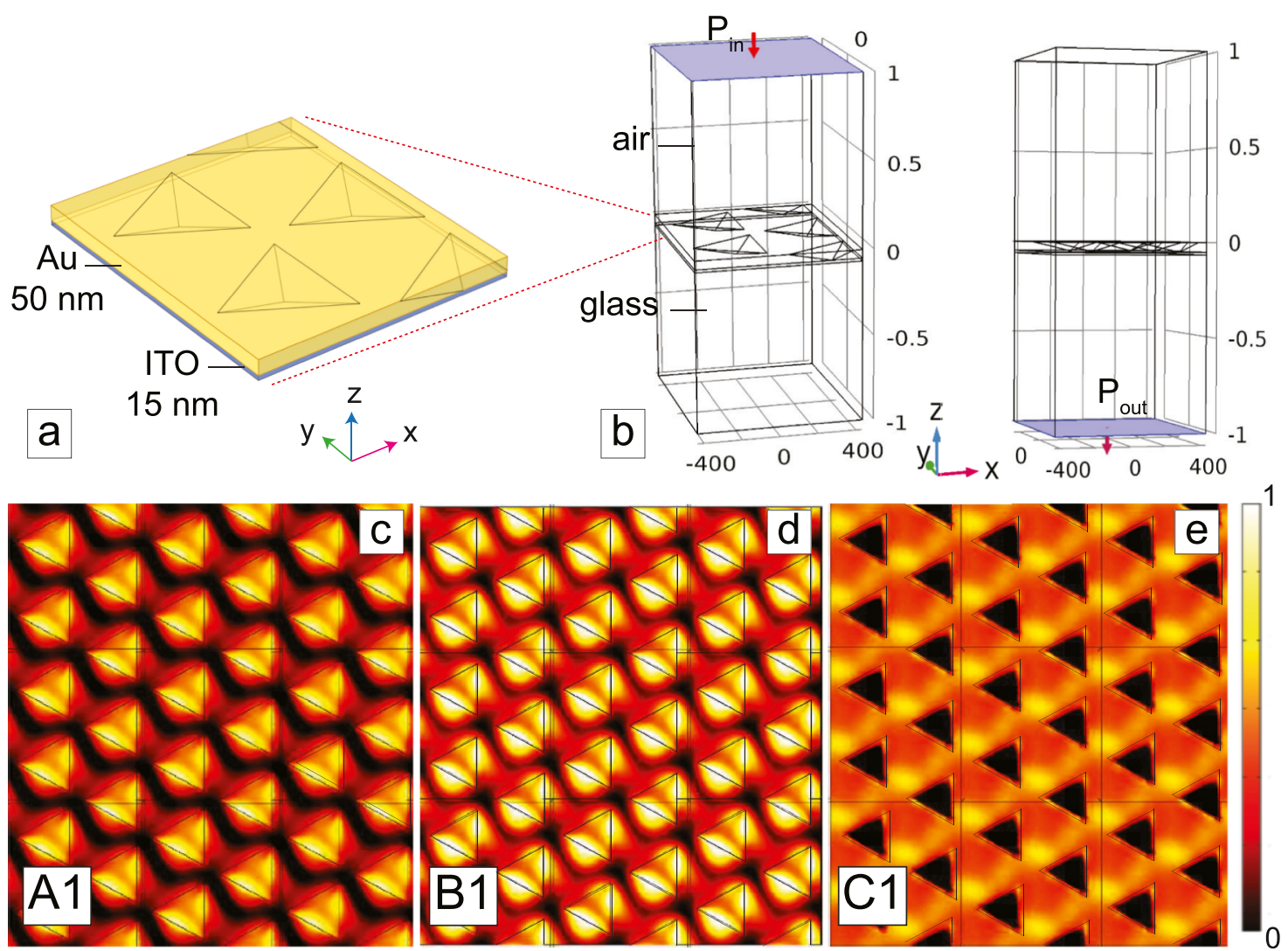

Figure 3. (a) Unit cell of the Au nanopyramid array simulated geometry. (b) COMSOL allows to simulate sources or detectors of EM radiation by creating ports. Simulated near-field enhancement maps $\left(|\mathrm{E}| / E_{0}\right)$ in the metasurface volume of samples (c) A1, (d) B1, and (e) C1 for a $45^{\circ}$ polarized $532 \mathrm{~nm}$ incident light beam.

absorbance, transmittance, and reflectance have been calculated for an Au layer taking the same value for the thickness of the metasurface and a silica metasurface displaying the same arrangement for the P-NHs and for the Au P-NHs metasurfaces, with a single $\mathrm{P}-\mathrm{NH}$ in the unit cell arranged in the same hexagonal template followed throughout this study (see the Supporting Information-Figure S3).

It is well known that the main feature of surface plasmon polaritons (SPPs), that is, cavity plasmon resonances and their coupling, arises in the near-field propagating regime. In fact, scanning near-field optical microscopy (SNOM) enables the opportunity to visualize the surface plasmon-mediated mechanism, as well as the enhanced transmission phenomenon, through the particular patterned structures, beyond the diffraction limit. ${ }^{52}$ To this end, a SNOM analysis has been carried out on the three samples by considering the typical excitation wavelength for gold $\left(\lambda_{\mathrm{exc}}=532 \mathrm{~nm}\right)$ at different incident polarizations. In addition to the typical $x$ and $y$ polarizations (not reported here), with respect to the P-NHs orientation, we considered the intermediate case of a $45^{\circ}$ polarization orientation.

Then, the transmitted signal through the three P-NHs has been acquired under the same experimental conditions (incident power and polarization of excitation laser source) in the SNOM-AFM combination mode. In this way, it is possible to obtain a topographic image with the corresponding SNOM analysis. Figure 2a,e,i reports the AFM topography of the three samples, obtained during the SNOM scan. The nearfield optical analysis of sample A1 is reported in Figure $2 b, c$ (Figure 2f,g for sample B1 and Figure 2j,k for sample C1). The three P-NH metasurfaces show the formation of electromagnetic hot spots in different regions of the samples. As we can see from the SNOM analysis, the interdistance among the P-NHs plays a fundamental role in the formation and localization of the hot spots on the metasurface. It is well known that the overall plasmonic modes observed on a metal film combine the surface plasmonic modes that propagate at the metal/dielectric interfaces on both sides of the excited film. In the presence of arrays of nanocavities, the SPP plasmonic modes that propagate on the surfaces can couple with the resonant modes localized in the cavity, confining part of the energy in it and intensifying the signal that is transmitted through $i^{52}$ or in the gap ${ }^{53,54}$ between the nanocavities, depending on the interdistance between the nanostructures.

This behavior is shown from sample A1 (Figure 2b,c), where the modes appear to be strongly confined inside the P-NHs, in particular on the edges of the nanocavities. This sample exhibits a minimal edge distance between the P-NHs and turns out to be the most efficient one in terms of the spatial confinement of the EM field when compared to the other two samples. This is made even more evident by extracting the line profiles from the topography and SNOM images (Figure 2d), with the maximum SNOM signal (photomultiplier tube, PMT counts in red) reaching close to the edge of the topographic line profile. A different situation is detected for sample B1, characterized by values of $d=160 \mathrm{~nm}$ and $a=550 \mathrm{~nm}$, increased by $50 \mathrm{~nm}$ compared to sample A1 (topography in Figure 2e). For this sample, the hot spots appear to be mainly confined inside the P-NHs, with a less intense near-field signal even in the gap regions between the nanocavities (Figure $2 \mathrm{f}-$ 

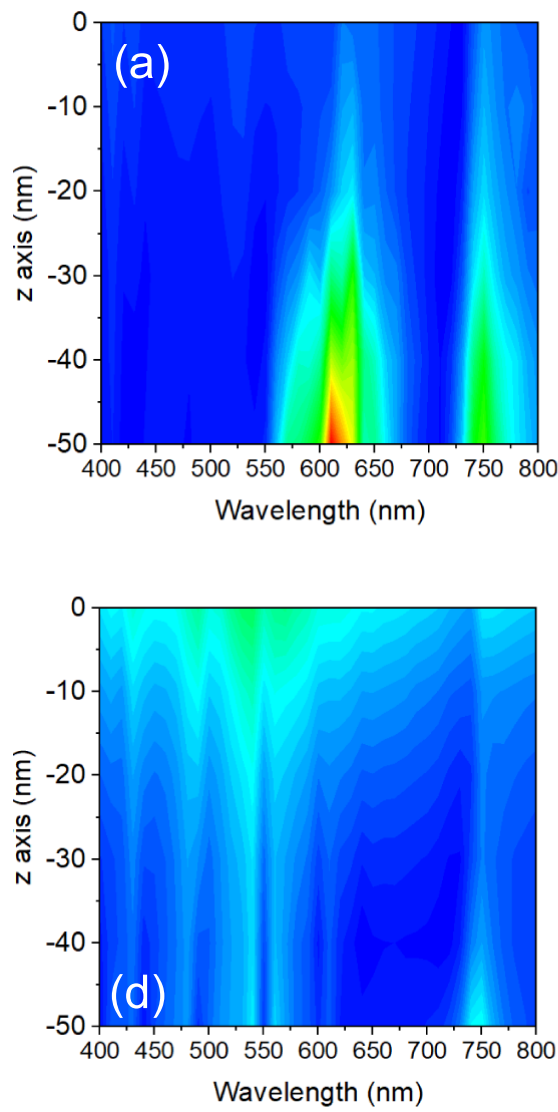
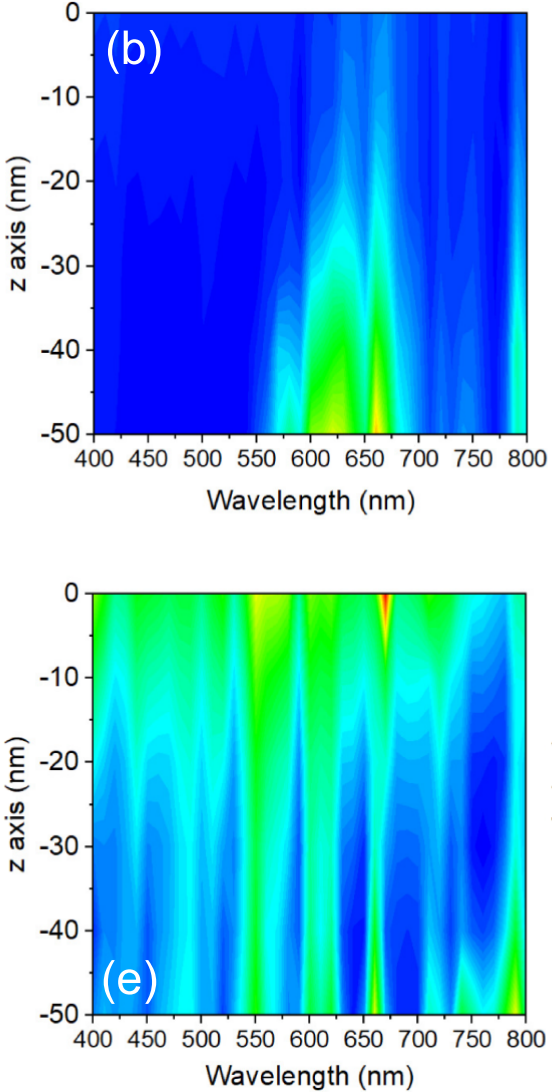

$|\mathrm{E}| / \mathrm{E}_{0}$

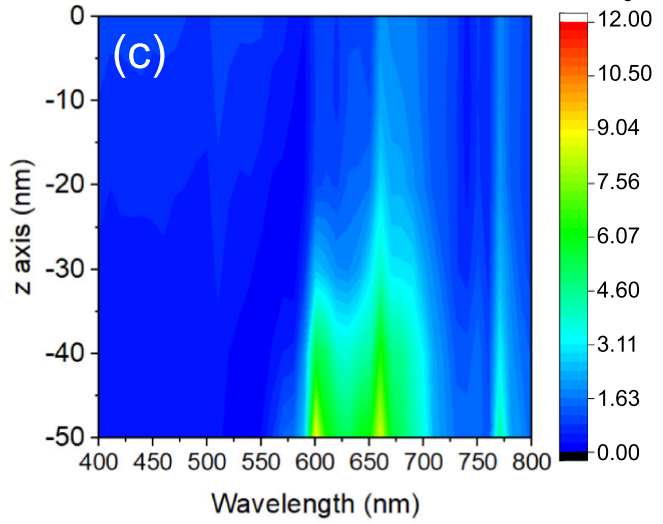

$\mathrm{E} / \mathrm{E}_{0}$

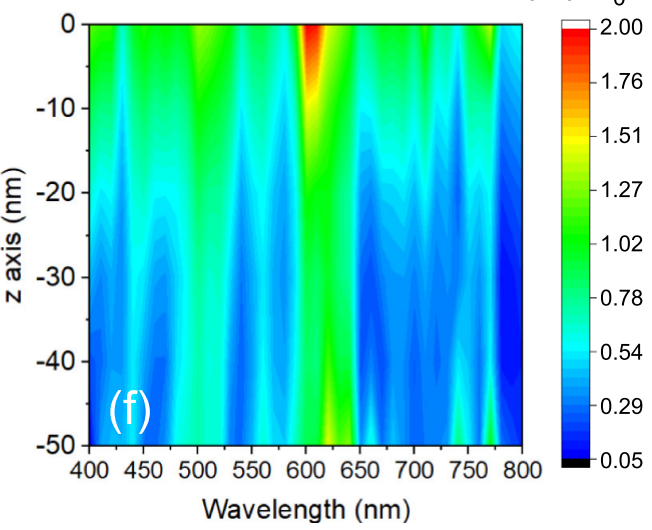

Figure 4. Electric field enhancement $|E| / E_{0}$ for samples $\mathrm{A} 1$ (a, d), B1 (b, e), and $\mathrm{C} 1(\mathrm{c}, \mathrm{f})$ as a function of the incident EM wave calculated in a point inside the P-NHs (top panels) and among the nanocavities (bottom panels) in the metasurface volume $(0<z<-50 \mathrm{~nm}, 0 \mathrm{~nm}$ correspond to the gold top surface).

h). A third and wholly different situation arises for sample $\mathrm{C} 1$ (Figure 2i, characterized by $d=250 \mathrm{~nm}$ and $a=640,90 \mathrm{~nm}$ more than sample B1): the near-field signal is then located totally out of the P-NHs and confined in the gaps in-between (Figure $2 \mathrm{j}, \mathrm{k}$ ). The line profiles of the topography and SNOM images (Figure 2l) confirm this behavior, with a maximum of the SNOM signal overlapping the gold gap region between the P-NHs. The SNOM analysis of the three samples reveals that a modulation of the metasurface optical response can be obtained by varying the distance between the nanocavities while keeping the dimensions of the P-NHs fixed. We can thus distinguish a first case in which the EM hot spots can be totally confined in the cavities (sample A1), a uniform distribution of the hot spots between the nanocavities and the gaps region (sample B1), and a third case in which the hot spots are totally confined in the gaps region (sample $\mathrm{C} 1$ ).

To further prove the significant role of the interdistance between the P-NHs in the development of metasurfaces with controlled EM hot spots, we performed numerical simulations based on a finite element method (FEM) on three different geometries representative of the samples analyzed experimentally. At this end, a unit cell is built in COMSOL Multiphysics and reported in Figure $3 \mathrm{a}, \mathrm{b}$. The simulated domain is characterized by periodic conditions on both sides of the parallelepiped to simulate an infinite array of the unit cell.

The software permits to simulate sources or detectors of EM radiation by creating ports: in our case, a port on the top $\left(\mathrm{P}_{\text {in }}\right)$ represents the input, from which the radiation starts to propagate, whereas a port on the bottom $\left(P_{\text {out }}\right)$, behaves as a detector (Figure $3 \mathrm{~b}$ ). For details related to the numerical analysis, see the "Numerical Simulations: Finite Element Method" in the Materials and Methods section. Figure 3c-e presents the $2 \mathrm{D}$ surface maps of the cumulative amplitude of the electric field $\left(|\mathbf{E}|=\sqrt{E_{x}^{2}+E_{y}^{2}+E_{z}^{2}}\right)$, normalized to the incident electric field $E_{0}$ in the entire metasurface volume (with $0<z<-50 \mathrm{~nm}, 0 \mathrm{~nm}$ corresponding to the gold top surface) for the three reported simulated geometries. As a result of the FEM analysis, it is possible to well distinguish bright modes inside each nanocavity for the simulated domain with geometrical parameters in accordance with sample A1, which moves in the edge regions and outside the nanocavities for the geometry corresponding to sample $\mathrm{B} 1$, while for the third geometry (corresponding to sample $\mathrm{C} 1$ ), it results in the confinement of hot spots within the nanocavities. Numerical simulations confirm the experimental results from the SNOM characterization.

It is well known that the field enhancement varies with the wavelength of the incident EM wave. Maps in Figure 4 obtained from a parametrized COMSOL simulation confirm this behavior for the three samples, considering two particular points on the metasurface: one located inside the P-NHs (referred to under "inside nanocavity") and the other between the nanocavities ("gap"). The analysis reports a normalized near-field enhancement of about 12 for sample A1 (the highest obtained value), obtained in the lower vertex of the pyramidal nanohole $(z=-50 \mathrm{~nm})$ and in the spectral range $610-640 \mathrm{~nm}$ 
(Figure 4a); a second band occurs in the range 740-780 nm. In the gap area between the nanocavities, there is no significant improvement of $|\mathbf{E}| / E_{0}$ in the considered spectral range (Figure $4 \mathrm{~d})$. For sample B1, the field enhancement is more significant in the range $575-685 \mathrm{~nm}$ with $|\mathrm{E}| / E_{0}$ values around $7-9$, with a second band around $785 \mathrm{~nm}$ (Figure 4b).

The satisfactory response of this sample over a wide spectral range in the gap region between the nanocavities (Figure $4 \mathrm{e}$ ) is noteworthy. For sample $C 1,|E| / E_{0}$ values are in a 6-7 interval in a 580-710 nm spectral range, while a good response was found for the gap region between the nanocavities (Figure $4 \mathrm{c}, \mathrm{f})$. Through this analysis, it is possible to identify the wavelength that best couples with the structure by exploiting both the interior of the P-NHs and the gaps in-between the nanocavities.

The SERS performances of the P-NHs were tested using the $785 \mathrm{~nm}$ excitation wavelength (Figure 5) to prove the

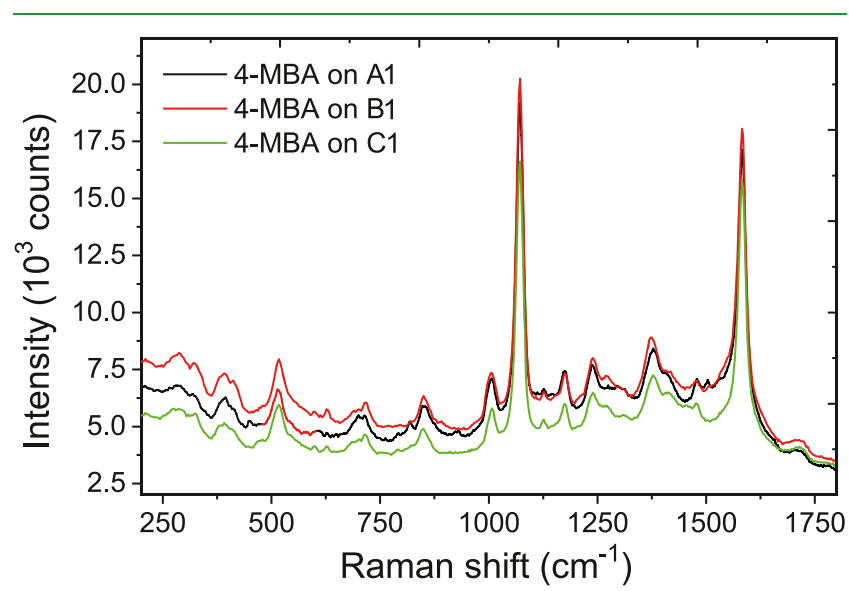

Figure 5. SERS spectrum of 4-mercaptobenzoic acid (4-MBA) probe on the plasmonic Au nanopyramid arrays A1, B1, and C1.

applicability of our metasurface for different SERS analyses. For a more sensitive detection of analytes at a low concentration, the structural tunability of the surface plasmon resonances of the nanoplatforms is an essential step to allow maximization of the SERS enhancement.

For this purpose, the SERS efficiency of our nanostructures with different edge-edge interdistance was investigated using a self-assembled monolayer (SAM) of the well-known Raman probe 4-mercaptobenzoic acid (4-MBA) (see Figure 5).
Furthermore, to evaluate the SERS enhancement ability of the designed plasmonic nanopatterns, the enhancement factor (EF) was calculated for the P-NHs from the spectral signal achieved of 4-MBA. EF values are shown to be $5.2 \times 10^{6}, 6 \times$ $10^{6}$, and $4.9 \times 10^{6}$ for samples $A 1, B 1$, and $C 1$, respectively. It reveals that all of the fabricated $\mathrm{P}-\mathrm{NH}$ substrates demonstrate a very strong SERS activity for effective Raman analysis, down to single-molecule detection. For details related to the EF calculation, see the "SERS Measurements" section and the related Supporting Information.

Figure 5 shows the SERS spectra of the Raman probe immobilized on the three nanostructures A1 (black line), B1 (red line), and C1 (green line). In all of the spectra, two main peaks of 4-MBA at 1073 and $1584 \mathrm{~cm}^{-1}$, associated with the CC stretching of the aromatic ring, can be clearly identified. The intensities of the three spectra fall in the same range as that of the higher value reached by nanostructure B1. This last result can be explained observing that for lower values of the edge-edge distance, the hot spot density characteristic of the nanostructures taken into account increases while the density of gold surface available for probe link decreases.

As a proof of concept, we used the geometry B1 to test the suitability of our metasurface for two different SERS analyses of virus. First of all, we proved that the naked substrate can be used to obtain the HAV SERS fingerprint. Next, we tested the possibility to realize a biosensor for quantitative HAV detection upon functionalization of the metasurface with an appropriate antibody. Figure 6a further shows the SERS spectrum fingerprint of the $\mathrm{HAV}$ virus in $10^{5} \mathrm{PFU} / \mathrm{mL}$ adsorbed on the plasmonic P-NH pattern B1. In the full spectrum, a predominant peak at $337 \mathrm{~cm}^{-1}$, associable to cysteine, is well visible.

The SERS spectrum of HAV (Figure 6a,b) can be characterized by typical features of nucleic acids, amino acids, and other biological components present in viruses. The strong peak at $525 \mathrm{~cm}^{-1}$, for example, can be attributed to the $S-S$ stretching mode of proteins. ${ }^{55}$ Spectral features of amino acids were found in the peak at $837 \mathrm{~cm}^{-1}$ (tyrosine)..$^{56,57}$ Spectral features of nucleic acids were found in the peaks at 677,1484 , and $1578 \mathrm{~cm}^{-1}$, assigned to guanine, ${ }^{58,59}$ and at 1232,1403 , and $1711 \mathrm{~cm}^{-1}$ assigned to uracil. $^{60}$ The band at $1127 \mathrm{~cm}^{-1}$ (C-N and $\mathrm{C}-\mathrm{C}$ stretches) is characteristic of the vibration of the proteins. ${ }^{56,57}$

Based on these encouraging steps, we proceeded to show that the metasurfaces studied here could also serve as SERS- (a)

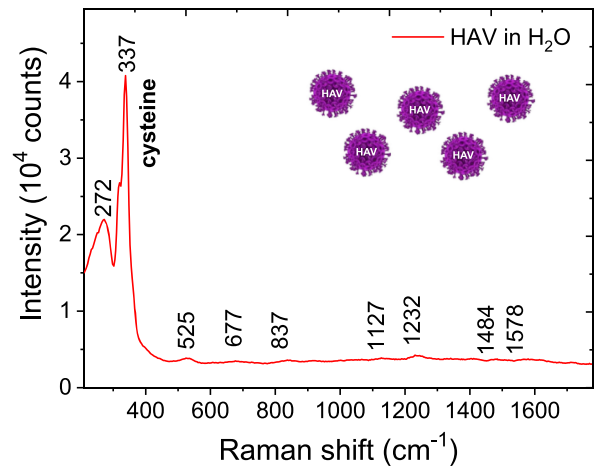

(b)

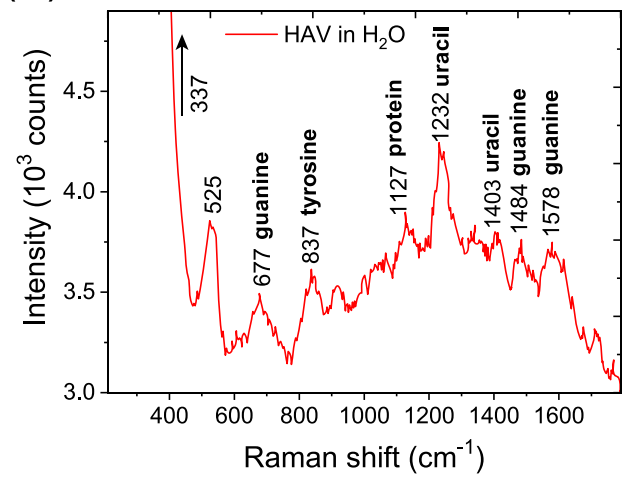

Figure 6. (a) SERS spectrum of the HAV virus in water $\left(10^{5} \mathrm{PFU} / \mathrm{mL}\right)$ adsorbed on the gold nanocavity pattern and (b) zooming on the peaks detected between 520 and $1600 \mathrm{~cm}^{-1}$ with tentative assignments to chemical groups present in the analyzed sample. 
(a)

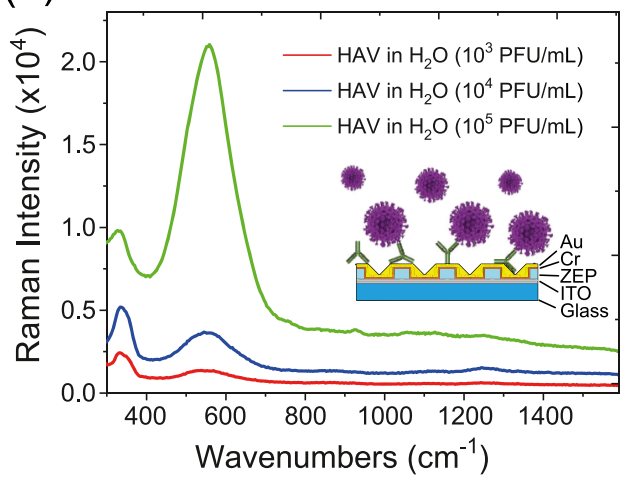

(b)

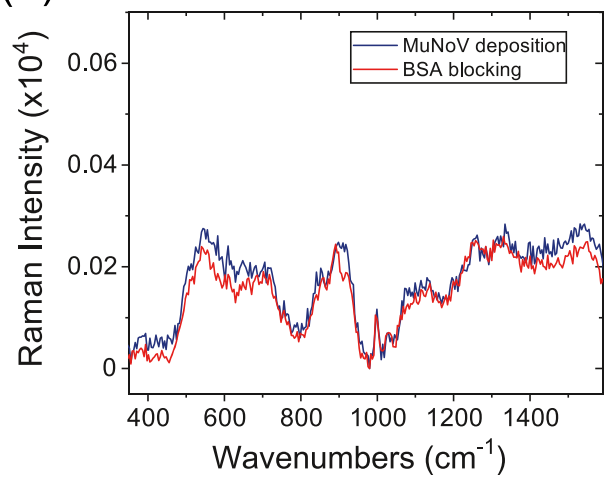

Figure 7. (a) SERS spectra of the $\mathrm{HAV}$ virus in $\mathrm{H}_{2} \mathrm{O}$ captured by its antibody [concentration $50 \mu \mathrm{g} / \mathrm{mL}$ in phosphate-buffered saline (PBS)], physically adsorbed on the nanostructure with a buffer of BSA at $3 \% \mathrm{w} / \mathrm{w}$, in concentrations of plaque-forming units of the virus of $10^{3} \mathrm{PFU} / \mathrm{mL}$ (red curve), $10^{4} \mathrm{PFU} / \mathrm{mL}$ (blue curve), and $10^{5} \mathrm{PFU} / \mathrm{mL}$ (green curve). The reference peak chosen on the basis of the evident amplification is that at $550 \mathrm{~cm}^{-1}$. (b) Detection specificity determined after the immunosensor incubation with $10^{5} \mathrm{PFU} / \mathrm{mL}$ of a nontarget virus, the Murine Norovirus $(\mathrm{MuNoV})$. The red curve refers to the spectra of the immunosensor after the BSA blocking, whereas the blue curve is acquired after the MuNoV deposition. No significant variations are appreciable between the two Raman traces.

based biosensors toward HAV detection. Upon physical adsorption of a monoclonal anti-HAV antibody onto the gold nanostructures and a surface passivation with $3 \% \mathrm{w} / \mathrm{w}$ bovine serum albumin (BSA) (see the "Virus Deposition for HAV SERS Fingerprint” section), extremely small volumes (2 $\mu \mathrm{L}$ ) of HAV target were dried on the functionalized surface. After substrate washing, HAV has been detected down to a concentration of $10^{3} \mathrm{PFU} / \mathrm{mL}$, corresponding to $\approx 13 \mathrm{pg} / \mathrm{mL}$ (further details are reported in the Supporting Information). Figure 7a shows the SERS spectra of the HAV in $\mathrm{H}_{2} \mathrm{O}$ captured by the antibody. In particular, we tested three different virus concentrations amounting to $10^{3} \mathrm{PFU} / \mathrm{mL}$ (red curve), $10^{4} \mathrm{PFU} / \mathrm{mL}$ (blue curve), and $10^{5} \mathrm{PFU} / \mathrm{mL}$ (green curve). Figure 7 a presents very strong SERS signals for all increasing concentrations.

Subsequently, the peak around $550 \mathrm{~cm}^{-1}$ (which can be assigned to $S-S$ stretching vibrations of disulfide bonds formed by cysteine $)^{56,57,61}$ can be considered a reference peak to detect the presence of the virus on the plasmonic substrate taken into account.

As a preliminary result, we referenced to the zero level all of the SERS spectra and integrated the peak at $550 \mathrm{~cm}^{-1}$ to evaluate the intensity enhancement due to the virus binding. We then plotted the $550 \mathrm{~cm}^{-1}$ peak areas vs the HAV concentrations to obtain a tentative calibration curve and evaluate a rough detection limit (DL) (see the Supporting Information). However, this can only be considered as a rough estimate, while more points will be needed to provide a robust and reliable DL. The error bars were inferred from the comparison of spectra obtained at three different locations on the metasurface and on three different replicas, pointing to a satisfactory reproducibility for our system. A preliminary DL value of $5260 \mathrm{PFU} / \mathrm{mL}(\approx 68 \mathrm{pg} / \mathrm{mL})$ was estimated from the residual standard deviation of the regression line. We believe that this result is interesting considering the literature. Indeed, to the best of our knowledge, only one work concerning the direct detection of the whole HAV virus has been published. In particular, Yang et al. described a resonance light-scattering sensor, with molecularly imprinted polymers as the recognition element, achieving a $\mathrm{DL}$ of $8.6 \mathrm{pmol} / \mathrm{L}(\approx 77 \mathrm{ng} / \mathrm{mL}) .^{38}$

In addition, the specificity of our immunofunctionalized nanostructure has been preliminarily verified in our control experiments using $10^{5} \mathrm{PFU} / \mathrm{mL} \mathrm{MuNoV}$ as the nontarget virus (Figure $7 \mathrm{~b}){ }^{62,63}$

Contrary to the spectra recorded during the HAV incubation onto the biofunctionalized substrate, in the case of MuNoV, no enhancement of the anti-HAV spectrum is observed. The antiHAV spectra recorded before and after the MuNoV incubation are practically identical, proving the lack of $\mathrm{MuNoV}$ capture by the antibody and therefore the specificity of our immunosurface (Figure 7b).

Our results open to the possibility to realize a plasmonic device for a sensitive detection of the HAV realized in a short time and in a label-free way that can be used for water analysis as a valid alternative to conventional methods.

\section{MATERIALS AND METHODS}

Nanostructure Fabrication and Morphological Characterization. We fabricated $300 \times 300 \mu \mathrm{m}^{2}$ Au nanostructures based on periodic arrays of P-NHs by using an EBL system (Raith 150). The P$\mathrm{NHs}$ are equilateral triangular based (side size $l=390 \mathrm{~nm}$ ) and arranged in a triangular geometry. We fabricated three patterns with the minimum interparticle distances $(d)$ of 110,160 , and $250 \mathrm{~nm}$. The nanostructures were fabricated realizing the conventional procedure of the EBL fabrication. A layer of an electron-sensitive resist (styrene methyl acrylate, ZEP 520 A) with a thickness of 180 $\mathrm{nm}$ was spin-coated on a glass substrate coated with $15 \mathrm{~nm}$ of indium tin oxide (ITO) and baked at $170^{\circ}$ for $5 \mathrm{~min}$. Then, it was exposed to the $13 \mathrm{pA}$ electron beam current with an area dose of $27 \mu \mathrm{C} / \mathrm{cm}^{2}$ to generate the desired geometry designed. The patterns made of P-NHs were achieved in the resist after development in an $n$-amyl acetate solvent and then rinsed for $60 \mathrm{~s}$ in a 1:3 methyl isobutyl ketone:isopropyl alcohol solution (MIBK:IPA) and for $30 \mathrm{~s}$ in IPA. Successively, on the resist surface, $2 \mathrm{~nm} \mathrm{Cr}$ and $50 \mathrm{~nm}$ Au films were evaporated using the SISTEC CL-400C e-beam system. Morphological characterization of the fabricated plasmonic nanostructures was performed using both scanning electron microscopy (SEMRaith 150) and atomic force microscopy (AFM) by Bioscope Catalyst in a contactless configuration using a silicon tip (radius $16 \mathrm{~nm}$ ).

Near-Field Characterization: SNOM Analysis. The near-field characterization was performed with a scanning near-field optical microscope (SNOM) alpha300 Sby WITec operating in the SNOMAFM combination mode. The transmitted signal through the three plasmonic inverted pyramidal metasurface samples has been acquired under the same experimental conditions (incident power and polarization of excitation laser source, $\lambda_{\text {exc }}=532 \mathrm{~nm}$ ). The laser beam is focused on the samples through an Al-coated aperture SNOM 
tip characterized by an aperture of lenght $60 \mathrm{~nm}$, while transmitted light is collected from the bottom by a $63 \times$ objective $(\mathrm{NA}=0.75)$ and detected by a photomultiplier tube (PMT).

Numerical Simulations: Finite Element Method. The calculation of optical properties of the inverted pyramidal metasurfaces has been performed by means of a FEM method, implemented with a commercial software (Comsol Multiphysics). For the calculation of near electric fields, an infinite array of plasmonic inverted nanopyramids have been considered. The unit cell is composed of a parallelepiped rectangle, characterized by air in the superstrate and glass in the substrate. In the middle, the metasurface is composed of a $50 \mathrm{~nm}$ thick Au layer in which the inverted tetrahedrons are arranged with the same orientations and distance of the samples. A TM electromagnetic wave at $532 \mathrm{~nm}$ propagating in the negative $z$-direction is used to excite the hot spots of the nanostructures.

Virus Deposition for HAV SERS Fingerprint. We carried out SERS measurements of the HAV physioadsorbed on the nanostructure with $a=50 \mathrm{~nm}$. Two microliters of HAV (HM175) in $\mathrm{mQ}_{2} \mathrm{O}$ with a concentration of $10^{5} \mathrm{PFU} / \mathrm{mL}$ was dropped on the surface of the gold nanopattern at room temperature. After $2 \mathrm{~h}$ of incubation, the $\mathrm{H}_{2} \mathrm{O}$ is completely evaporated, leaving only the virus on the substrate. The substrate was washed with both phosphate-buffered saline (PBS) and deionized water to remove the unadsorbed virus and then tested with the SERS tool.

Substrate Biofunctionalization and Virus Deposition for Immuno-SERS. We functionalized the nanopattern B1 with edgeedge distance $160 \mathrm{~nm}$ to realize a potentially specific SERS sensor. The functionalization process is realized using $50 \mu \mathrm{g} / \mathrm{mL}$ of a mouse monoclonal anti-HAV antibody (IgG3HepA(1886) from Santa Cruz Biotechnology). After $12 \mathrm{~h}$ of incubation, the substrates were washed as in the physioadsorbed case to remove the unfixed antibody. Subsequently, the nonspecific adsorption sites on the surface of the substrate were passivated with $10 \mu \mathrm{L}$ of bovine serum albumin (BSA) blocking solution (3\% BSA in PBS) for $1 \mathrm{~h}$ at room temperature, and the substrate was washed again with PBS and deionized water to remove the residual BSA. The functionalized nanopatterns were SERS tested to detect $2 \mu \mathrm{L}$ of $\mathrm{HAV}$ in $\mathrm{mQ} \mathrm{H}_{2} \mathrm{O}$ with different concentrations ranging from $10^{3}$ to $10^{5} \mathrm{PFU} / \mathrm{mL}$. After virus deposition, the solvent was left to evaporate for $\approx 15 \mathrm{~min}$, and then, the samples were kept in aerobic conditions for further $30 \mathrm{~min}$ to promote immunobonding before washing with $m Q \mathrm{H}_{2} \mathrm{O}$. We repeated the virus deposition procedure on new immunofunctionalized patterns using $10^{5} \mathrm{PFU} / \mathrm{mL}$ Murine Norovirus (MuNoV, MNV1) to test by SERS measurements the specificity of our system. In our experiments, the virus suspensions were deposited in a laboratory with biosafety level 2 (BSL-2, c/o Istituto Zooprofilattico del Mezzogiorno, Portici, Italy).

SERS Measurements. SERS analysis of the HAV was performed using a QE Pro-Raman spectrophotometer (Ocean Optics) coupled with an upright microscope Olympus BX51 in a backscattering configuration. The system was configured for $\lambda=785 \mathrm{~nm}(12 \mathrm{~mW})$, with a grating of 1200 lines $/ \mathrm{mm}$ and an input slit of $50 \mu \mathrm{m}$. The spectra were collected in the range $200-1800 \mathrm{~cm}^{-1}$, with $10 \mathrm{~s}$ of acquisition time, a $50 \times(\mathrm{NA}=0.75)$ microscope objective, and a laser spot with a diameter of about $20 \mu \mathrm{m}$. In general, we reported an average spectrum for each tested sample. Mean spectra were calculated from at least three repeated measurements on different points of the metasurface and on three different replicas.

The enhancement factor (EF) of the plasmonic nanopatterns designed was measured evaluating the spectral signal achieved for a self-assembled monolayer (SAM) of 4-mercaptobenzoic acid (4MBA), a molecule probe widely used in the literature to test SERS substrate. The SAM was obtained by submerging the nanostructures for $12 \mathrm{~h}$ in a $100 \mu \mathrm{M}$ ethanolic solution of 4-MBA at room temperature. Subsequently, the surface was washed with water and ethanol to remove any excess of the probe molecule noncovalently bound onto gold. The EF of the nanopatterns with three different interparticle distances was evaluated as described in a previous work. ${ }^{12}$ In particular, for this evaluation, we used the following well- known equation: $E_{\mathrm{F}}=\left(I_{\mathrm{s}} \times N_{\mathrm{r}}\right) /\left(I_{\mathrm{r}} \times N_{\mathrm{s}}\right)$, where $I_{\mathrm{s}}$ and $I_{\mathrm{r}}$ are respectively the integrated intensities of the main SERS peaks at 1073 $\mathrm{cm}^{-1}$ of 4-MBA molecules adsorbed on the different substrates and 4MBA powder, while $N_{\mathrm{s}}$ and $N_{\mathrm{r}}$ are the number of 4-MBA molecules contributing to the signal in the two cases considered at the irradiation spot of the laser, respectively.

\section{CONCLUSIONS}

In this work, we proposed a novel plasmonic nanostructure based on high-density metallic inverted pyramidal nanohole arrays fabricated by combining a top-down process EBL and thin metallic film deposition technologies that showed remarkable sensitivity performances and enhanced electromagnetic fields. As a proof of concept of the potentialities of our nanosensor in virus detection, an extremely small volume $(2 \mu \mathrm{L})$ of HAV target was dried on the functionalized array, and HAV has been detected at a concentration of $13 \mathrm{pg} / \mathrm{mL}$. This new tool has the potential to provide results over short times and can be used in field or in laboratories without adequate instrumental resources for biomolecular techniques.

\section{ASSOCIATED CONTENT}

\section{(s) Supporting Information}

The Supporting Information is available free of charge at https://pubs.acs.org/doi/10.1021/acsami.1c12525.

Morphological profile of a single Au nanocavity; far-field characterization: Vis-NIR spectroscopy; comparison of the optical response of the considered hexagonal arrangement $\mathrm{P}-\mathrm{NH}$ metasurface and other geometries; EF calculation and SERS measurements; minimal HAV concentration detected; calibration curve and detection limit; and comparison of the pyramidal nanohole metasurface with the commercial Klarite sensors (PDF)

\section{AUTHOR INFORMATION}

\section{Corresponding Authors}

Antonio De Luca - Department of Physics, University of Calabria, Via P. Bucci, 87036 Rende, CS, Italy; CNR NANOTEC-Istituto di Nanotecnologia, UOS Cosenza, 87036 Rende, CS, Italy; O orcid.org/0000-0003-24289075; Email: antonio.deluca@unical.it

Lucia Petti - Institute of Applied Sciences and Intelligent Systems "E. Caianiello" CNR, 80078 Pozzuoli, Italy; Email: 1.petti@isasi.cnr.it

\section{Authors}

Giovanna Palermo - Department of Physics, University of Calabria, Via P. Bucci, 87036 Rende, CS, Italy; CNR NANOTEC-Istituto di Nanotecnologia, UOS Cosenza, 87036 Rende, CS, Italy; ○ orcid.org/0000-0001-5649$735 \mathrm{X}$

Massimo Rippa - Institute of Applied Sciences and Intelligent Systems "E. Caianiello" CNR, 80078 Pozzuoli, Italy; (1) orcid.org/0000-0002-1993-4589

Ylli Conti - Department of Physics, University of Calabria, Via P. Bucci, 87036 Rende, CS, Italy

Ambra Vestri - Institute of Applied Sciences and Intelligent Systems "E. Caianiello" CNR, 80078 Pozzuoli, Italy

Riccardo Castagna - Institute of Applied Sciences and Intelligent Systems "E. Caianiello" CNR, 80078 Pozzuoli, Italy 
Giovanna Fusco - Department of Food Safety, Nutrition and Veterinary Public Health, Istituto Superiore di Sanitá, 00161 Rome, Italy

Elisabetta Suffredini - Department of Food Safety, Nutrition and Veterinary Public Health, Istituto Superiore di Sanitá, 00161 Rome, Italy

Jun Zhou - Institute of Photonics, Faculty of Science, Ningbo University, 315211 Ningbo, People's Republic of China

Joseph Zyss - LUMIN Laboratory (CNRS), Institut d'Alembert, Universitè Paris Saclay, 91190 Gif sur Yvette, France

Complete contact information is available at: https://pubs.acs.org/10.1021/acsami.1c12525

\section{Author Contributions}

${ }^{\nabla}$ G.P. and M.R. contributed equally to this work.

\section{Notes}

The authors declare no competing financial interest.

\section{ACKNOWLEDGMENTS}

The authors gratefully acknowledge the support for this work by MIUR funding the project "Design and development of environmental sensors for the research of microbiological and chemical contaminants hazardous to health (H2O Safety)" in the framework of Fund for Development and Cohesion (FSC) Proof of Concept projects (D.D. 07/06/19 prot. no. 1096, POC01 00109) and European Regional Development Fund-FESR of POR Campania 2014-2020-projects for the technological transfer and first industrialization of highpotential innovative companies for the fight against oncological pathologies-Campania Terra del Buono Program (Project title: Multiplex nanostructured platform for label-free detection of food-borne pathogens and carcinogenic pesticides (MultiPath)). The authors thank Dr. Eugenia Bobeico from ENEA Portici center for the gold evaporation on the nanostructures. Furthermore, this research has been supported by the "AIM: Attraction and International Mobility"-PON R\&I 20142020 Calabria. The authors thank the Area della Ricerca di Roma 2, Tor Vergata, for the access to the ICT Services (ARToV-CNR) and the use of the COMSOL Multiphysics Platform and Origin Lab, and the Infrastructure BeyondNano (PONa3-00362) of CNR-Nanotec for the access to research instruments.

\section{REFERENCES}

(1) Wang, Y.; Yan, B.; Chen, L. SERS Tags: Novel Optical Nanoprobes for Bioanalysis. Chem. Rev. 2013, 113, 1391-1428.

(2) Su, X.; Wang, Y.; Wang, W.; Sun, K.; Chen, L. Phospholipid Encapsulated AuNR@Ag/Au Nanosphere SERS Tags with Environmental Stimulus Responsive Signal Property. ACS Appl. Mater. Interfaces 2016, 8, 10201-10211.

(3) Korkmaz, A.; Kenton, M.; Aksin, G.; Kahraman, M.; Wachsmann-Hogiu, S. Inexpensive and Flexible SERS Substrates on Adhesive Tape Based on Biosilica Plasmonic Nanocomposites. ACS Appl. Nano Mater. 2018, 1, 5316-5326.

(4) Yang, K.; Wang, J.; Yao, X.; Lyu, D.; Zhu, J.; Yang, Z.; Liu, B.; Ren, B. Large-Area Plasmonic Metamaterial with Thickness-Dependent Absorption. Adv. Opt. Mater. 2021, 9, No. 2001375.

(5) Saviñon-Flores, F.; Méndez, E.; López-Castaños, M.; CarabarinLima, A.; López-Castaños, K. A.; González-Fuentes, M. A.; MéndezAlbores, A. A Review on SERS-Based Detection of Human Virus Infections: Influenza and Coronavirus. Biosensors 2021, 11, No. 66.

(6) Trojanowicz, R. K.; Matczyszyn, K.; Castagna, R.; Rippa, M.; Bobeico, E.; Zhou, J.; Petti, L. Real-Time Surface-Enhanced Raman
Scattering Tracking of Adenine-Gold Charge Transfer Complex Formation on Nanocavity-Shaped Plasmonic Crystals. J. Phys. Chem. C 2019, 123, 17961-17967.

(7) Cao, C.; Zhang, J.; Wen, X.; Dodson, S. L.; Dao, N. T.; Wong, L. M.; Wang, S.; Li, S.; Phan, A. T.; Xiong, Q. Metamaterials-Based Label-Free Nanosensor for Conformation and Affinity Biosensing. ACS Nano 2013, 7, 7583-7591.

(8) Lio, G. E.; Ferraro, A.; Ritacco, T.; Aceti, D. M.; De Luca, A.; Giocondo, M.; Caputo, R. Leveraging on ENZ Metamaterials to Achieve 2D and 3D Hyper-Resolution in Two-Photon Direct Laser Writing. Adv. Mater. 2021, 33, No. 2008644.

(9) Jáuregui-López, I.; Rodríguez-Ulibarri, P.; Kuznetsov, S. A.; Quemada, C.; Beruete, M. Labyrinth Metasurface for Biosensing Applications: Numerical Study on the New Paradigm of Metageometries. Sensors 2019, 19, No. 4396.

(10) Zhang, Z.; Yang, M.; Yan, X.; Guo, X.; Li, J.; Yang, Y.; Wei, D.; Liu, L.; Xie, J.; Liu, Y. The Antibody-Free Recognition of Cancer Cells Using Plasmonic Biosensor Platforms with the Anisotropic Resonant Metasurfaces. ACS Appl. Mater. Interfaces 2020, 12, 11388 11396.

(11) Rippa, M.; Castagna, R.; Pannico, M.; Musto, P.; Bobeico, E.; Zhou, J.; Petti, L. High-Performance Nanocavities-Based MetaCrystals for Enhanced Plasmonic Sensing. Opt. Data Process. Storage 2016, 2, 22-29.

(12) Rippa, M.; Castagna, R.; Pannico, M.; Musto, P.; Bobeico, E.; Zhou, J.; Petti, L. Plasmonic Nanocavities-Based Aperiodic Crystal for Protein-Protein Recognition SERS Sensors. Opt. Data Process. Storage 2017, 3, 54-60.

(13) Rippa, M.; Castagna, R.; Tkachenko, V.; Zhou, J.; Petti, L. Engineered Nanopatterned Substrates for High-Sensitive Localized Surface Plasmon Resonance: An Assay on Biomacromolecules. J. Mater. Chem. B 2017, 5, 5473-5478.

(14) Palermo, G.; Sreekanth, K. V.; Maccaferri, N.; Lio, G. E.; Nicoletta, G.; De Angelis, F.; Hinczewski, M.; Strangi, G. Hyperbolic Dispersion Metasurfaces for Molecular Biosensing. Nanophotonics 2021, 10, 295-231.

(15) Palermo, G.; Lio, G. E.; Esposito, M.; Ricciardi, L.; Manoccio, M.; Tasco, V.; Passaseo, A.; De Luca, A.; Strangi, G. Biomolecular Sensing at the Interface Between Chiral Metasurfaces and Hyperbolic Metamaterials. ACS Appl. Mater. Interfaces 2020, 12, 3018-30188.

(16) Sreekanth, K. V.; Alapan, Y.; ElKabbash, M.; Ilker, E.; Hinczewski, M.; Gurkan, U. A.; De Luca, A.; Strangi, G. Extreme Sensitivity Biosensing Platform Based on Hyperbolic Metamaterials. Nat. Mater. 2016, 15, 621-627.

(17) Sreekanth, K. V.; Ouyang, Q.; Sreejith, S.; Zeng, S.; Lishu, W.; Ilker, E.; Dong, W.; ElKabbash, M.; Ting, Y.; Lim, C. T.; Hinczewski, M.; Strangi, G.; Yong, K.-T.; Simpson, R. E.; Singh, R. Phase-ChangeMaterial-Based Low-Loss Visible-Frequency Hyperbolic Metamaterials for Ultrasensitive Label-Free Biosensing. Adv. Opt. Mater. 2019, 7, No. 1900081.

(18) Sánchez, G.; Bosch, A.; Pintó, R. Hepatitis a Virus Detection in Food: Current and Future Prospects. Lett. Appl. Microbiol. 2007, 45, $1-5$.

(19) Choi, Y.-H.; Lee, G.-Y.; Ko, H.; Chang, Y. W.; Kang, M.-J.; Pyun, J.-C. Development of SPR Biosensor for the Detection of Human Hepatitis B Virus Using Plasma-Treated Parylene-N Film. Biosens. Bioelectron. 2014, 56, 286-294.

(20) Lepage, D.; Jiménez, A.; Beauvais, J.; Dubowski, J. J. Real-Time Detection of Influenza a Virus Using Semiconductor Nanophotonics. Light: Sci. Appl. 2013, 2, No. e62.

(21) Bai, H.; Wang, R.; Hargis, B.; Lu, H.; Li, Y. A SPR Aptasensor for Detection of Avian Influenza Virus H5N1. Sensors 2012, 12, 12506-12518.

(22) Rippa, M.; Castagna, R.; Brandi, S.; Fusco, G.; Monini, M.; Chen, D.; Zhou, J.; Zyss, J.; Petti, L. Octupolar Plasmonic Nanosensor Based on Ordered Arrays of Triangular Au Nanopillars for Selective Rotavirus Detection. ACS Appl. Nano Mater. 2020, 3, 4837-4844. 
(23) Rippa, M.; Castagna, R.; Pannico, M.; Musto, P.; Borriello, G.; Paradiso, R.; Galiero, G.; Bolletti Censi, S.; Zhou, J.; Zyss, J.; Petti, L. Octupolar Metastructures for a Highly Sensitive, Rapid, and Reproducible Phage-Based Detection of Bacterial Pathogens by Surface-Enhanced Raman Scattering. ACS Sens. 2017, 2, 947-954.

(24) Yan, H.; Peng, B.; He, W.; Zhong, G.; Qi, Y.; Ren, B.; Gao, Z.; Jing, Z.; Song, M.; Xu, G.; Sui, J.; Li, W. Molecular Determinants of Hepatitis B and D Virus Entry Restriction in Mouse Sodium Taurocholate Cotransporting Polypeptide. J. Virol. 2013, 87, 79777991.

(25) Shanmukh, S.; Jones, L.; Driskell, J.; Zhao, Y.; Dluhy, R.; Tripp, R. A. Rapid and Sensitive Detection of Respiratory Virus Molecular Signatures Using a Silver Nanorod Array SERS Substrate. Nano Lett. 2006, 6, 2630-2636.

(26) Mauriz, E. Recent Progress in Plasmonic Biosensing Schemes for Virus Detection. Sensors 2020, 20, No. 4745.

(27) Alexander, T. A. Development of Methodology Based on Commercialized SERS-Active Substrates for Rapid Discrimination of Poxviridae Virions. Anal. Chem. 2008, 80, 2817-2825.

(28) Park, J.-E.; Kim, K.; Jung, Y.; Kim, J.-H.; Nam, J.-M. Metal Nanoparticles for Virus Detection. ChemNanoMat 2016, 2, 927-936.

(29) Driskell, J. D.; Zhu, Y.; Kirkwood, C. D.; Zhao, Y.; Dluhy, R. A.; Tripp, R. A. Rapid and Sensitive Detection of Rotavirus Molecular Signatures Using Surface Enhanced Raman Spectroscopy. PLoS One 2010, 5, No. e10222.

(30) Dotzauer, A. Hepatitis A Virus. In Encyclopedia of Virology; Elsevier, 2008; Vol. 19, pp 343-350.

(31) Gossner, C.; Severi, E. Three Simultaneous, Food-Borne, MultiCountry Outbreaks of Hepatitis a Virus Infection Reported in EPISFWD in 2013: What Does It Mean for the European Union? Eurosurveillance 2014, 19, No. 20941.

(32) Petrignani, M.; Verhoef, L.; Vennema, H.; van Hunen, R.; Baas, D.; van Steenbergen, J. E.; Koopmans, M. P. Underdiagnosis of Foodborne Hepatitis A, the Netherlands, 2008-2010. Emerging Infect. Dis. 2014, 20, No. 596.

(33) Bonomi, A. E.; Patrick, D. L.; Bushnell, D. M.; Martin, M. Validation of the United States' Version of the World Health Organization Quality of Life (WHOQOL) Instrument. J. Clin. Epidemiol. 2000, 53, 1-12.

(34) Neethirajan, S.; Ahmed, S. R.; Chand, R.; Buozis, J.; Nagy, É. Recent Advances in Biosensor Development for Foodborne Virus Detection. Nanotheranostics 2017, 1, No. 272.

(35) Sánchez, G. Hepatitis a Virus in Food: Detection and Inactivation Methods; Springer, 2013; pp 9-20.

(36) Bazzardi, R.; Fattaccio, M. C.; Salza, S.; Canu, A.; Marongiu, E.; Pisanu, M. Preliminary Study on Norovirus, Hepatitis a Virus, Escherichia coli and Their Potential Seasonality in Shellfish from Different Growing and Harvesting Areas in Sardinia Region. Ital. J. Food Saf. 2014, 3, No. 1601.

(37) Manzano, M.; Viezzi, S.; Mazerat, S.; Marks, R. S.; Vidic, J. Rapid and Label-Free Electrochemical DNa Biosensor for Detecting Hepatitis a Virus. Biosens. Bioelectron. 2018, 100, 89-95.

(38) Yang, B.; Gong, H.; Chen, C.; Chen, X.; Cai, C. A Virus Resonance Light Scattering Sensor Based on Mussel-Inspired Molecularly Imprinted Polymers for High Sensitive and High Selective Detection of Hepatitis a Virus. Biosens. Bioelectron. 2017, $87,679-685$.

(39) Cao, Y. C.; Jin, R.; Mirkin, C. A. Nanoparticles with Raman Spectroscopic Fingerprints for DNa and RNa Detection. Science 2002, 297, 1536-1540.

(40) Jang, K.-J.; Lee, H.; Jin, H.-L.; Park, Y.; Nam, J.-M. RestrictionEnzyme-Coded Gold-Nanoparticle Probes for Multiplexed DNa Detection. Small 2009, 5, 2665-2668.

(41) Liu, Y.; Fang, W.; Wu, Z.; Zhou, G.; Yi, W.; Zhou, X.; Shen, A.; $\mathrm{Hu}$, J. A One-Tube Multiplexed Colorimetric Strategy Based on Plasmonic Nanoparticles Combined with Non-Negative Matrix Factorization. Talanta 2014, 128, 305-310.

(42) Su, J.; Wang, D.; Nörbel, L.; Shen, J.; Zhao, Z.; Dou, Y.; Peng, T.; Shi, J.; Mathur, S.; Fan, C.; Song, S. Multicolor Gold-Silver
Nano-Mushrooms As Ready-To-Use SERS Probes for Ultrasensitive and Multiplex DNA/miRNa Detection. Anal. Chem. 2017, 89, 25312538.

(43) Sun, C.-H.; Min, W.-L.; Linn, N. C.; Jiang, P.; Jiang, B. Templated Fabrication of Large Area Subwavelength Antireflection Gratings on Silicon. Appl. Phys. Lett. 2007, 91, No. 231105.

(44) Mavrokefalos, A.; Han, S. E.; Yerci, S.; Branham, M. S.; Chen, G. Efficient Light Trapping in Inverted Nanopyramid Thin Crystalline Silicon Membranes for Solar Cell Applications. Nano Lett. 2012, 12, 2792-2796.

(45) Ray, K.; Lakowicz, J. R. Metal-Enhanced Fluorescence Lifetime Imaging and Spectroscopy on a Modified SERS Substrate. J. Phys. Chem. C 2013, 117, 15790-15797.

(46) Botti, S.; Cantarini, L.; Almaviva, S.; Puiu, A.; Rufoloni, A. Assessment of SERS Activity and Enhancement Factors for Highly Sensitive Gold Coated Substrates Probed with Explosive Molecules. Chem. Phys. Lett. 2014, 592, 277-281.

(47) Liszewska, M.; Bartosewicz, B.; Budner, B.; Nasiłowska, B.; Szala, M.; Weyher, J. L.; Dzięcielewski, I.; Mierczyk, Z.; Jankiewicz, B. J. Evaluation of Selected SERS Substrates for Trace Detection of Explosive Materials Using Portable Raman Systems. Vib. Spectrosc. 2019, 100, 79-85.

(48) Kolkowski, R.; Szeszko, J.; Dwir, B.; Kapon, E.; Zyss, J. Effects of Surface Plasmon Polariton-Mediated Interactions on Second Harmonic Generation from Assemblies of Pyramidal Metallic NanoCavities. Opt. Express 2014, 22, 30592-30606.

(49) Kolkowski, R.; Szeszko, J.; Dwir, B.; Kapon, E.; Zyss, J. NonCentrosymmetric Plasmonic Crystals for Second-Harmonic Generation with Controlled Anisotropy and Enhancement. Laser Photonics Rev. 2016, 10, 287-298.

(50) Haramoto, E.; Kitajima, M.; Hata, A.; Torrey, J. R.; Masago, Y.; Sano, D.; Katayama, H. A Review on Recent Progress in the Detection Methods and Prevalence of Human Enteric Viruses in Water. Water Res. 2018, 135, 168-186.

(51) Fusco, G.; Anastasio, A.; Kingsley, D. H.; Amoroso, M. G.; Pepe, T.; Fratamico, P. M.; Cioffi, B.; Rossi, R.; La Rosa, G.; Boccia, F. Detection of Hepatitis a Virus and Other Enteric Viruses in Shellfish Collected in the Gulf of Naples, Italy. Int. J. Environ. Res. Public Health 2019, 16, No. 2588.

(52) Dhama, R.; Caligiuri, V.; Petti, L.; Rashed, A. R.; Rippa, M.; Lento, R.; Termine, R.; Caglayan, H.; De Luca, A. Extraordinary Effects in Quasi-Periodic Gold Nanocavities: Enhanced Transmission and Polarization Control of Cavity Modes. ACS Nano 2018, 12, 504512.

(53) Xue, X.; Fan, Y.; Segal, E.; Wang, W.; Yang, F.; Wang, Y.; Zhao, F.; Fu, W.; Ling, Y.; Salomon, A.; Zhengjun, Z. Periodical Concentration of Surface Plasmon Polaritons by Wave Interference in Metallic Film with Nanocavity Array. Mater. Today 2021, 46, 5461.

(54) Salomon, A.; Prior, Y.; Fedoruk, M.; Feldmann, J.; Kolkowski, R.; Zyss, J. Plasmonic Coupling Between Metallic Nanocavities. J. Opt. 2014, 16, No. 114012.

(55) Stremersch, S.; Marro, M.; Pinchasik, B.-E.; Baatsen, P.; Hendrix, A.; De Smedt, S. C.; Loza-Alvarez, P.; Skirtach, A. G.; Raemdonck, K.; Braeckmans, K. Identification of Individual Exosomelike Vesicles by Surface Enhanced Raman Spectroscopy. Small 2016, 12, 3292-3301.

(56) Szekeres, G. P.; Kneipp, J. SERS Probing of Proteins in Gold Nanoparticle Agglomerates. Front. Chem. 2019, 7, No. 30.

(57) Rygula, A.; Majzner, K.; Marzec, K. M.; Kaczor, A.; Pilarczyk, M.; Baranska, M. Raman Spectroscopy of Proteins: A Review. J. Raman Spectrosc. 2013, 44, 1061-1076.

(58) Xu, L.-J.; Lei, Z.-C.; Li, J.; Zong, C.; Yang, C. J.; Ren, B. LabelFree Surface-Enhanced Raman Spectroscopy Detection of DNa with Single-Base Sensitivity. J. Am. Chem. Soc. 2015, 137, 5149-5154.

(59) Willets, K. A. Surface-Enhanced Raman Scattering (SERS) for Probing Internal Cellular Structure and Dynamics. Anal. Bioanal. Chem. 2009, 394, 85-94. 
(60) Madzharova, F.; Heiner, Z.; Gühlke, M.; Kneipp, J. SurfaceEnhanced Hyper-Raman Spectra of Adenine, Guanine, Cytosine, Thymine, and Uracil. J. Phys. Chem. C 2016, 120, 15415-15423.

(61) Fazio, B.; D’Andrea, C.; Foti, A.; Messina, E.; Irrera, A.; Donato, M. G.; Villari, V.; Micali, N.; Maragò, O. M.; Gucciardi, P. G. SERS Detection of Biomolecules at Physiological PH Via Aggregation of Gold Nanorods Mediated by Optical Forces and Plasmonic Heating. Sci. Rep. 2016, 6, No. 26952.

(62) De Paula, V.; Gaspar, A.; Villar, L. Optimization of Methods for Detecting Hepatitis a Virus in Food. Food Environ. Virol. 2010, 2, 4752.

(63) Zeipe, G. Picornaviruses. In Textbook of Medical Virology; Butterworths, 1983; Vol. 59, pp 242-250. 